Trauma Berufskrankh 2012 · 14[Suppl 2]:125-134 DOI 10.1007/s10039-012-1861-1

Online publiziert: 24. Mai 2012

(c) Springer-Verlag 2012

T. Pohlemann · P. Mörsdorf · U. Culemann · A. Pizanis

Klinik für Unfall-, Hand- und Wiederherstellungschirurgie,

Universitätsklinikum des Saarlandes, Homburg/Saar

\title{
Behandlungsstrategie bei Azetabulumfraktur
}

soll. Aufgrund der zunehmenden Relevanz von Azetabulumfrakturen bei geriatrischen Patienten werden auch zu dieser Thematik neue Ansätze angesprochen.

Beckenring- und Azetabulumfrakturen unterscheiden sich in der Regel durch ihre Bedrohlichkeit im Hinblick auf den Gesamtzustand des Patienten. Während Beckenringfrakturen auch heutzutage noch häufig lebensbedrohliche Zustände erzeugen, handelt es sich bei Azetabulumfrakturen oft um isolierte Verletzungen, bei denen die Behandlung der vitalen Bedrohung eher in den Hintergrund tritt. Jedoch stellt die zeitnahe Entscheidungsfindung über eine operative Therapie einen entscheidenden Faktor bei diesen Verletzungen dar. Ziel ist, so schnell als möglich eine weitestgehend komplett anatomische Wiederherstellung der Gelenkfläche zu gewährleisten, da ansonsten die Gefahr einer Früharthrose schnell ansteigt.

Während bei Beckenringfrakturen v. a. die Menge des Blutverlusts, der Zustand der Weichteile, das Ausmaß der Begleitverletzungen und die Stabilität des Beckenringes die entscheidende Rolle spielen und damit die Notwendigkeit für Notfallmaßnahmen und den zeitlichen $\mathrm{Ab}$ lauf der Eingriffe festlegen, sind die Prioritäten bei Azetabulumfrakturen anders gelagert. Ihr Management konzentriert sich zunächst auf

- das Erkennen des spezifischen Frakturverlaufs,

- die Klassifikation,

- die Feststellung des Ausmaßes eventueller Knorpelschäden sowie

- die möglichst exakte Planung der operativen Versorgung.

Bei Letzterer spielen neben der allgemeinen Infrastruktur und der Erfahrung des behandelnden Teams natürlich auch der Operationszeitpunkt, die vorhandenen Instrumente und Implantate, die Operationstechnik und nicht zuletzt die eigene operative Erfahrung eine entscheidende Rolle. realität. Aufgrund der komplizierten anatomischen Verhältnisse, der oft schwierigen Röntgenbildinterpretation und der schlechten operativen Erreichbarkeit des Azetabulums entwickelte sich die operative Behandlung, insbesondere komplizierterer Azetabulumfrakturen, zwischenzeitlich zu einem unfallchirurgischen Spezialgebiet. Die Vermittlung von Kenntnissen ist langwierig, auch theoretische Einführungskurse umfassen in der Regel mehrere Tage, sodass in dem hier gegebenen Rahmen lediglich die Wertigkeit der Klassifikation auf die Entscheidungsfindung und operative Technik dargestellt werden

\begin{tabular}{|c|c|c|c|}
\hline Verletzung & $\begin{array}{l}\text { Konservativ be- } \\
\text { handelte Patienten }\end{array}$ & $\begin{array}{l}\text { Operativ be- } \\
\text { handelte Patienten }\end{array}$ & $\begin{array}{l}\text { Insgesamt be- } \\
\text { handelte Patienten }\end{array}$ \\
\hline Beckenring $\mathrm{A}$ & 220 & 11 & 231 \\
\hline Beckenring $\mathrm{B}$ & 50 & 107 & 157 \\
\hline Beckenring C & 4 & 186 & 190 \\
\hline Azetabulum & 39 & 213 & 252 \\
\hline Azetabulum und Becken & 5 & 21 & 26 \\
\hline Spätkorrekturen & 29 Beckenring & 7 Azetabulum & 36 \\
\hline Summe & 347 & 545 & 892 \\
\hline
\end{tabular}




\section{Becken, Acetabulum, hüftgelenksnahe Frakturen}
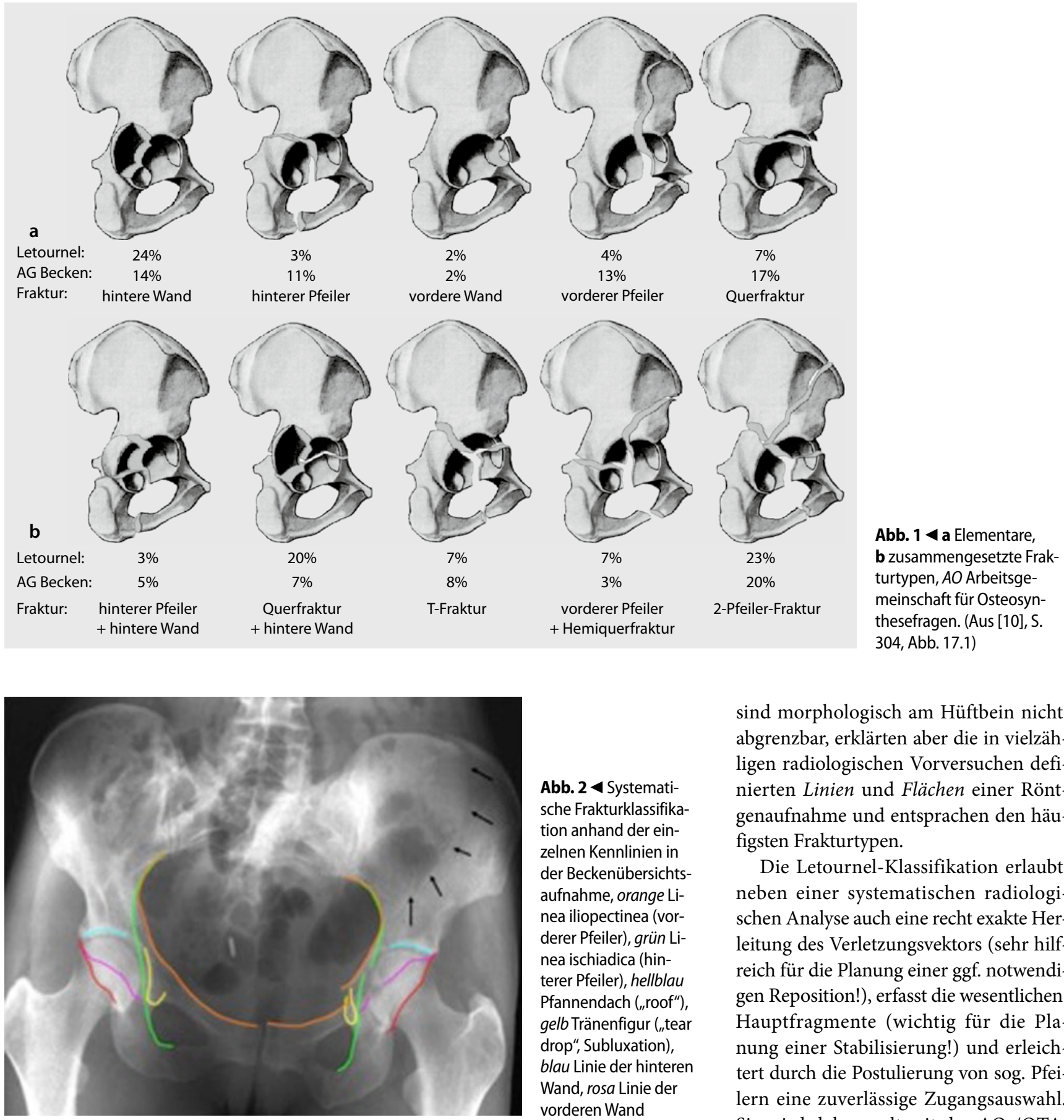

Abb. $2<$ Systematische Frakturklassifikation anhand der einzelnen Kennlinien in der Beckenübersichtsaufnahme, orange Linea iliopectinea (vorderer Pfeiler), grün Linea ischiadica (hinterer Pfeiler), hellblau Pfannendach (,"roof"), gelb Tränenfigur (,tear drop", Subluxation), blau Linie der hinteren Wand, rosa Linie der vorderen Wand

\section{Klassifikation der Azetabulumfrakturen}

Derzeit sind sowohl die in der 1960er Jahren veröffentlichte Klassifikation von Letournel [4] und Judet et al. [5] sowie die 1996 veröffentlichte die AO-/OTA-Klassifikationen (AO: Arbeitsgemeinschaft für Osteosynthesefragen, OTA: „Orthopedic Trauma Association“) für Azetabulumfrakturen gebräuchlich [7].

\section{Vor- und Nachteile der einzelnen Klassifikationen}

Grundlegende Untersuchungen zur Morphologie, der Verletzungsmechanik und der Röntgenanatomie waren die Grundlage zur Erstellung der Letournel-Klassifikation. Sie führten zunächst zur Beschreibung eines sog. vorderen und hinteren Pfeilers mit dazwischen liegender azetabularer Gelenkfläche. Diese Strukturen sind morphologisch am Hüftbein nicht abgrenzbar, erklärten aber die in vielzähligen radiologischen Vorversuchen definierten Linien und Flächen einer Röntgenaufnahme und entsprachen den häufigsten Frakturtypen.

Die Letournel-Klassifikation erlaubt neben einer systematischen radiologischen Analyse auch eine recht exakte Herleitung des Verletzungsvektors (sehr hilfreich für die Planung einer ggf. notwendigen Reposition!), erfasst die wesentlichen Hauptfragmente (wichtig für die Planung einer Stabilisierung!) und erleichtert durch die Postulierung von sog. Pfeilern eine zuverlässige Zugangsauswahl. Sie wird daher weltweit der AO-/OTAKlassifikation vorgezogen. Letztere versucht durch eine Gliederung in 3 Gruppen sich der allgemeinen Systematik der AO-Klassifikation anzunähern, basiert aber ebenfalls auf den 2 Hauptgruppen des Letournel-Systems (einfache oder elementare und zusammengesetzte Frakturtypen). Die in der AO-Klassifikation künstlich gebildete Gruppe der C-Frakturen (entspricht den 2-Pfeiler-Frakturen der Letournel-Klassifikation) weist trotz radiologischer Kompliziertheit in der kli- 
nischen Erfahrung auch noch eine bessere Prognose als die wesentlich schwerer zu rekonstruierenden B-Frakturen auf. Da auch retrospektiv eine ggf. notwendige Umklassifizierung in die AO-Klassifikation problemlos gelingt, sollte auf alle Fälle zur Primärevaluation, Entscheidungsfindung und Operationsplanung die Klassifikation nach Letournel eingesetzt werden. Sie unterscheidet in 2 Hauptgruppen 10 Frakturtypen:

Elementare Frakturtypen. Sie zeichnen sich durch eine einzige Frakturlinie aus. Unterschieden werden dabei

- die posterioren Typen (Fraktur der hinteren Wand/Fraktur des hinteren Pfeilers) von den

- anterioren Frakturtypen (Fraktur der vorderen Wand/Fraktur des vorderen Pfeilers). Da der vordere Pfeiler das gesamte Ilium umfassen kann, sind diese Bruchverletzungen oft schwer zu identifizieren.

Eine Besonderheit in der elementaren Gruppe stellt die Querfraktur dar, die zwar beide Pfeiler betrifft, aber ebenfalls nur eine einzelne Frakturlinie umfasst (• Abb. 1a).

Zusammengesetzte Frakturtypen. In dieser Gruppe werden die empirisch am häufigsten Frakturkombinationen in weitere 5 Typen unterschieden ( $\mathbf{A b b}$. 1b). Es gibt

- die Fraktur der hinteren Wand mit hinterem Pfeiler,

- die Querfraktur mit Fraktur der hinteren Wand,

- die Fraktur des vorderen Pfeilers mit hinterer Hemiquerfraktur,

- die T-Fraktur (eigentlich Querfraktur mit distaler Separation von vorderem und hinterem Pfeiler) und

- die herausgehobene komplette Fraktur beider Pfeiler, kurz 2-Pfeiler-Fraktur.

Letztere ist definiert durch eine komplette Abtrennung aller Gelenk tragenden Fragmente vom Stammskelett. Diese hohe Instabilität hat aber auch Vorteile, da sich durch Ablagerung der Fragmente um die neue Kopfposition eine sog. prognos-

Trauma Berufskrankh 2012 · 14[Suppl 2]:125-134 DOI 10.1007/s10039-012-1861-1

c) Springer-Verlag 2012

\section{T. Pohlemann · P. Mörsdorf $\cdot$ U. Culemann · A. Pizanis}

\section{Behandlungsstrategie bei Azetabulumfraktur}

\section{Zusammenfassung}

Die differenzierte Therapie von Azetabulumfrakturen ist mittlerweile ein Spezialgebiet der Unfallchirurgie. Basierend auf den grundlegenden Untersuchungen von Letournel und später Matta konnte eine ganz wesentliche Standardisierung von Diagnostik, Klassifikation, Entscheidungsfindung sowie operativen Möglichkeiten bei diesen Verletzungen erreicht werden. Da eine exakte Wiederherstellung der anatomischen Gelenkgeometrie für die behinderungsfreie Ausheilung essenziell ist, ist die exakte Kenntnis der Verfahrensweise zur Diagnostik, der Interpretation der bildgebenden Verfahren sowie der Frakturklassifikation notwendig, um eine hochqualitative Versorgung, ggf. auch Ent- scheidungsfindung zur Weiterverlegung in ein Spezialzentrum, zu gewährleisten. Aufgrund der immer älter werdenden Bevölkerung stellen Azetabulumfrakturen bei Patienten mit verminderter Knochenqualität ein zunehmendes Problem dar. Während diese Fälle noch vor 20 Jahren als Kontraindikation für eine operative Therapie galten, kann durch modifizierte Techniken inzwischen auch in der Altersgruppe über 65 Jahre in etwa 80\% der Fälle ein gutes Langzeitergebnis durch Gelenkrekonstruktion erreicht werden.

\section{Schlüsselwörter}

Azetabulum · Knochenfrakturen .

Rekonstruktion · Standardisierung · Geriatrie

\section{Treatment strategies for acetabular fractures}

\section{Abstract}

The operative treatment of acetabular fractures has meanwhile developed into a subspeciality in trauma surgery. The primary evaluation, classification, indication, and the approaches are still based on the gold standard inaugurated by Letournel and Judet and further developed by Matta and colleagues. Because exact anatomical reconstruction of the joint's geometry is essential for disability-free outcome, exact knowledge of the differential diagnosis, interpretation of images, and fracture classification is mandatory in order to provide high-quality care and, if necessary, refer the patient to a specialized center.
Due to the rapidly rising number of geriatric patients, acetabular fractures in patients with reduced bone quality represent an increasing challenge. Whereas 20 years ago, reduced bone quality was a contraindication to surgery, modified techniques can in the meantime also be used in the age group 65 years and over, with good long-term results from joint reconstruction being achieved in approximately $80 \%$ of cases.

\section{Keywords}

Acetabulum - Bone fractures - Reconstruction . Standardization $\cdot$ Geriatrics tisch günstige sekundäre Kongruenz ausbilden kann.

$\mathrm{Zu}$ weiteren Details und ggf. notwendigen Verfeinerungen der Klassifikationen wird auf die entsprechende Literatur verwiesen $[4,5,8]$.

\section{Klassifikation der Azetabulumfrakturen}

Sie erfolgt anhand einer Beckenübersichtsaufnahme und je einer Ala- und Obturatoraufnahme. Das Becken wird dabei jeweils rechts oder links etwas $40^{\circ}$ angehoben. Es wird empfohlen, diese Schrägaufnahmen ebenfalls als Übersichtsaufnahmen anzufertigen, da die unverletzte
Seite so als komplementäre Vergleichsaufnahme zur Verfügung steht.

Die Analyse erfolgt durch systematische Identifikation der definierten Kennlinien auf der verletzten und unverletzten Seite sowie die Akquise von radiologischen Zusatzinformationen ( $\bullet$ Abb. 2). Auch diesbezüglich sei auf die Primärliteratur verwiesen.

In unserem eigenen Vorgehen bewährte sich ein Ausschlussverfahren (- Abb. 3). Aus den 10 Möglichkeiten werden zunächst alle unmöglichen Frakturtypen ausgeschlossen. Beispielsweise schließt eine intakte Kennlinie des vorderen Pfeilers (Linea iliopectinea) das Vorliegen einer Fraktur des vorderen Pfei- 


\section{Becken, Acetabulum, hüftgelenksnahe Frakturen}

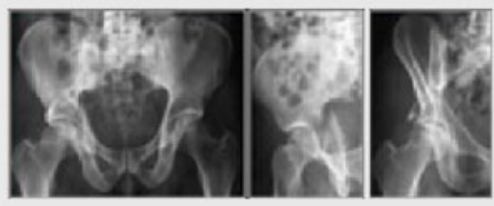

1.L.iliopectinea unterbrochen

2. L.ilioischiadica unterbrochen

3. Foramen obturatum unverletzt

4. Fraktur hintere Wand??

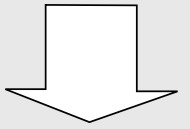

Querfraktur mit hinterer Wand
Ausschlussdiagnostik aus

den 10 Fx Typen:
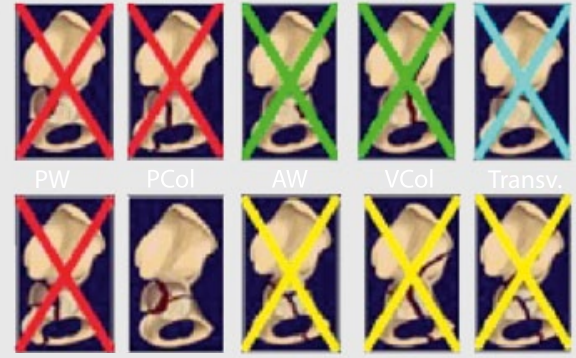

$\mathrm{Tr}+\mathrm{PW}$

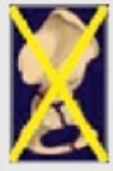

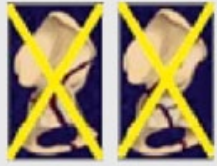

Both C.
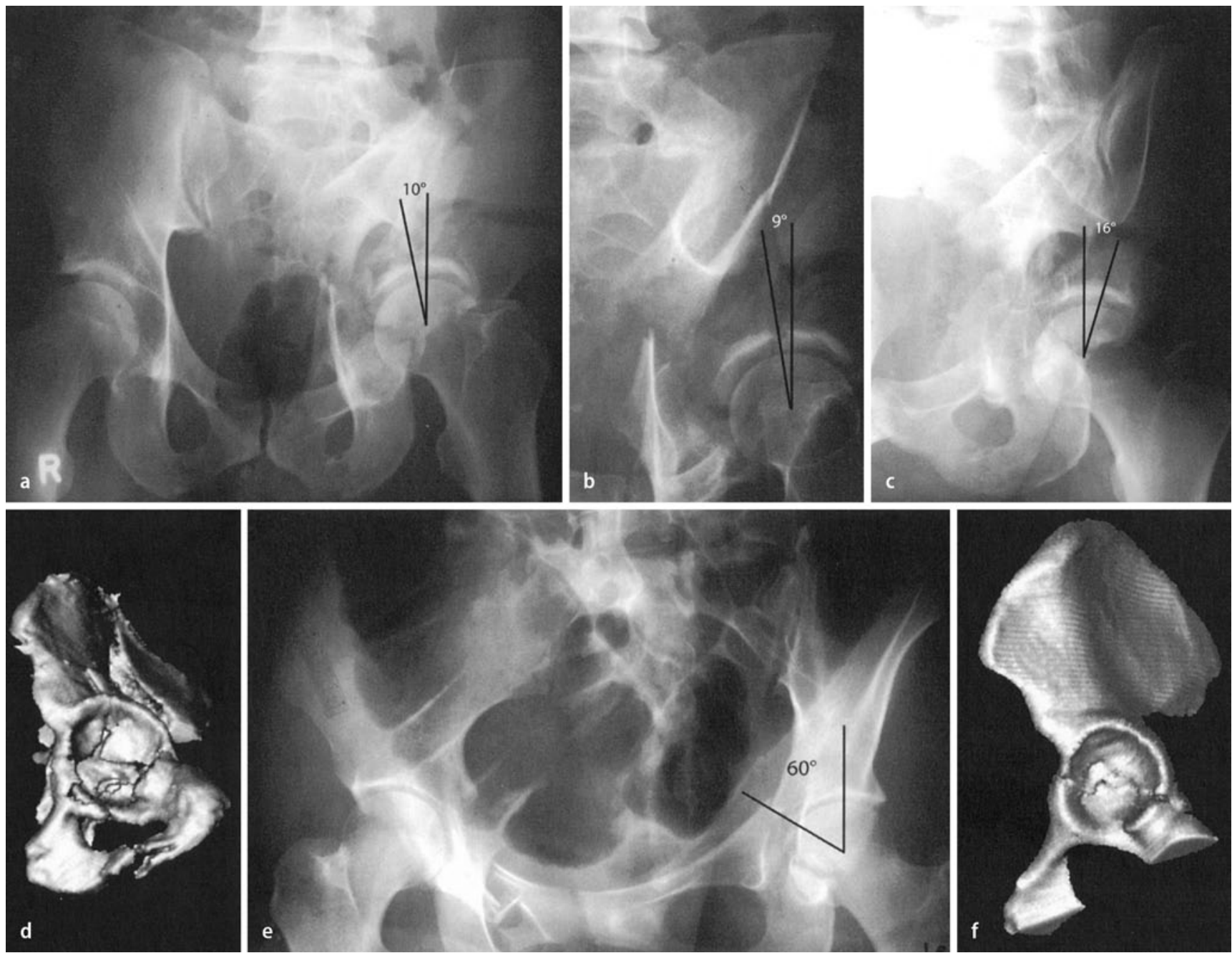

Abb. 4 வ Dachwinkelmessungen nach Azetabulumfrakturen: Bestimmung des Pfannendachwinkels nach Matta et al. [6]: a-d in der a.-p. (a), Ala- (b) und Obturatorprojektion (c) sowie Verdeutlichung im dreidimensionalen Femursubtraktionscomputertomogramm (d); $\mathbf{e}$,f weiteres Beispiel: Pfannendachwinkel in allen 3 Projektionen $>45^{\circ}$ : bei zentrierter stabiler Hüfte konservative Therapie indiziert. (Aus [10], Kap. 18, Abb. 18.2., Abb. 18.3) 

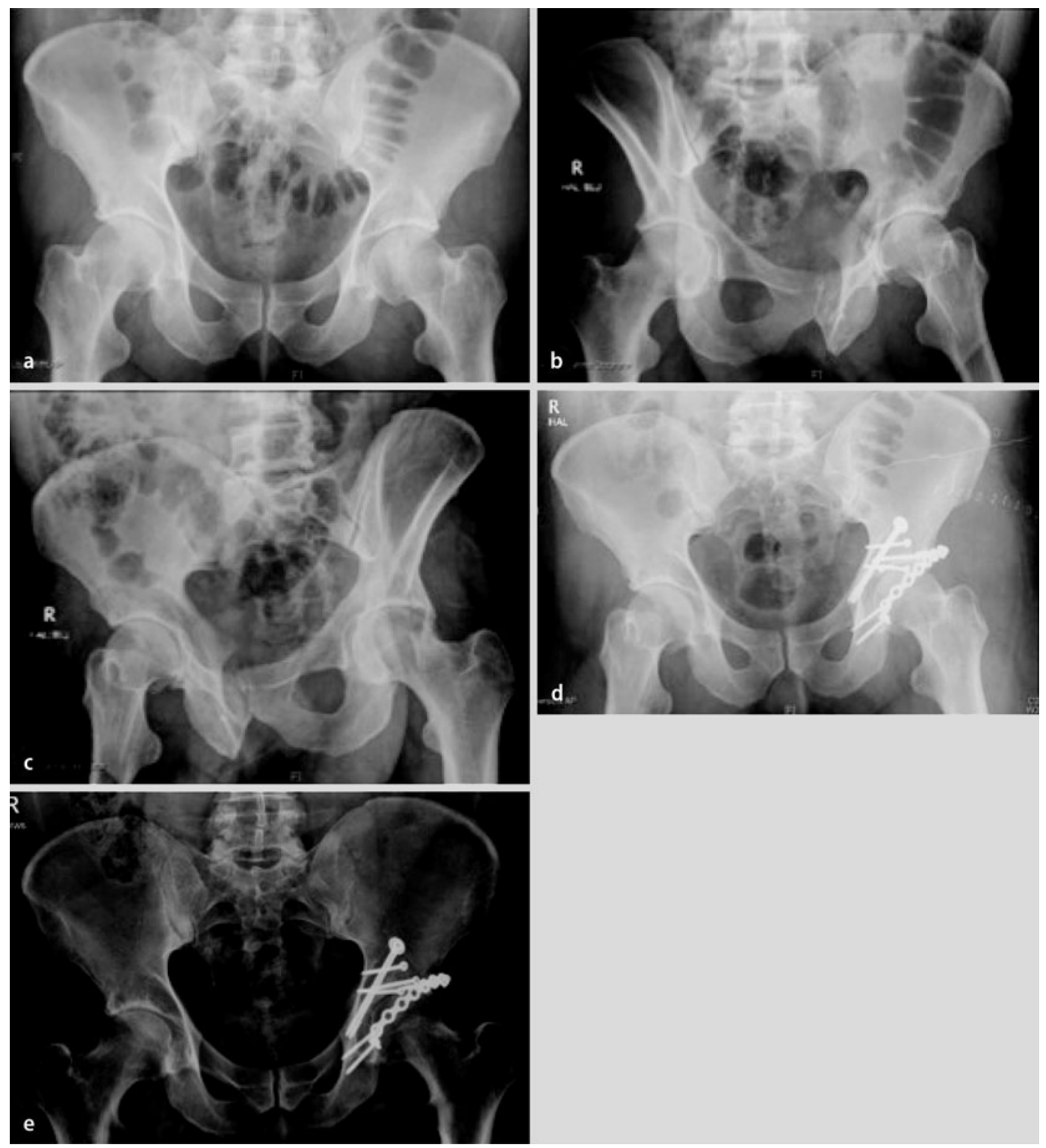

Abb. $5 \varangle$ Fallbeispiel 1, a-c Verletzung präoperativ (Querfraktur und hintere Wand), d Reposition über Kocher-LangenbeckZugang, e Befund nach 2 Jahren

lers, einer vorderen Wand, einer Querfraktur, einer T-Fraktur, einer Querfraktur mit hinterer Wand und einer 2-Pfeiler-Fraktur aus. Mit wenigen Aussagen lässt sich so schnell und zuverlässig eine Eingrenzung vornehmen, bei der allenfalls noch ähnliche Frakturtypen unterschieden werden müssen und auf alle Fälle eine Entscheidungsfindung herbeigeführt werden kann.

\section{Operative Versorgung}

\section{Indikation}

Die Indikation orientiert sich weiterhin an den Kriterien

- Gelenkkongruenz,
- Gelenkstabilität,

- azetabulare Zusatzverletzungen und

- Allgemeinzustand des Patienten.

Zur Abschätzung der Gelenkstabilität ist die Messung der Pfannendachwinkel nach Matta et al. [6] weiterhin ein gutes, einfaches Hilfsmittel, um die Größe des verbliebenen Pfannendachfragments abzuschätzen. In allen 3 Standardröntgenaufnahmen werden das Zentrum des Hüftgelenks bestimmt, eine Senkrechte angelegt und der Winkel bis zur ersten erkennbaren Frakturlinie gemessen. Ist dieser in allen 3 Projektionen größer als $40^{\circ}$, kann bei bestehender Gelenkkongruenz auch bei konservativer Therapie von einer guten Prognose ausgegangen werden (• Abb.4).

Die CT-Diagnostik (CT: Computertomographie) ist zwischenzeitlich zur Detailanalyse obligat, in der Regel als feinschichtiges Spiral-CT. Dabei sollte das ganze Becken inklusive Sakroiliakalgelenk und Tuber ischiadicum dargestellt werden, denn nur so ist eine systematische Analyse möglich, die einerseits stabile und instabile Fragmente analysiert und Zusatzverletzungen aufdeckt (Gelenkimpression, intraartikuläre Fragmente, Trümmerzonen usw.). 3D-Reformationen dienen im Wesentlichen zur Zusammenfassung der Befunde und zur Planung von Reposition und Stabilisierung. Sie als alleinige Analyse zu nutzen, ist auf- 


\section{Becken, Acetabulum, hüftgelenksnahe Frakturen}
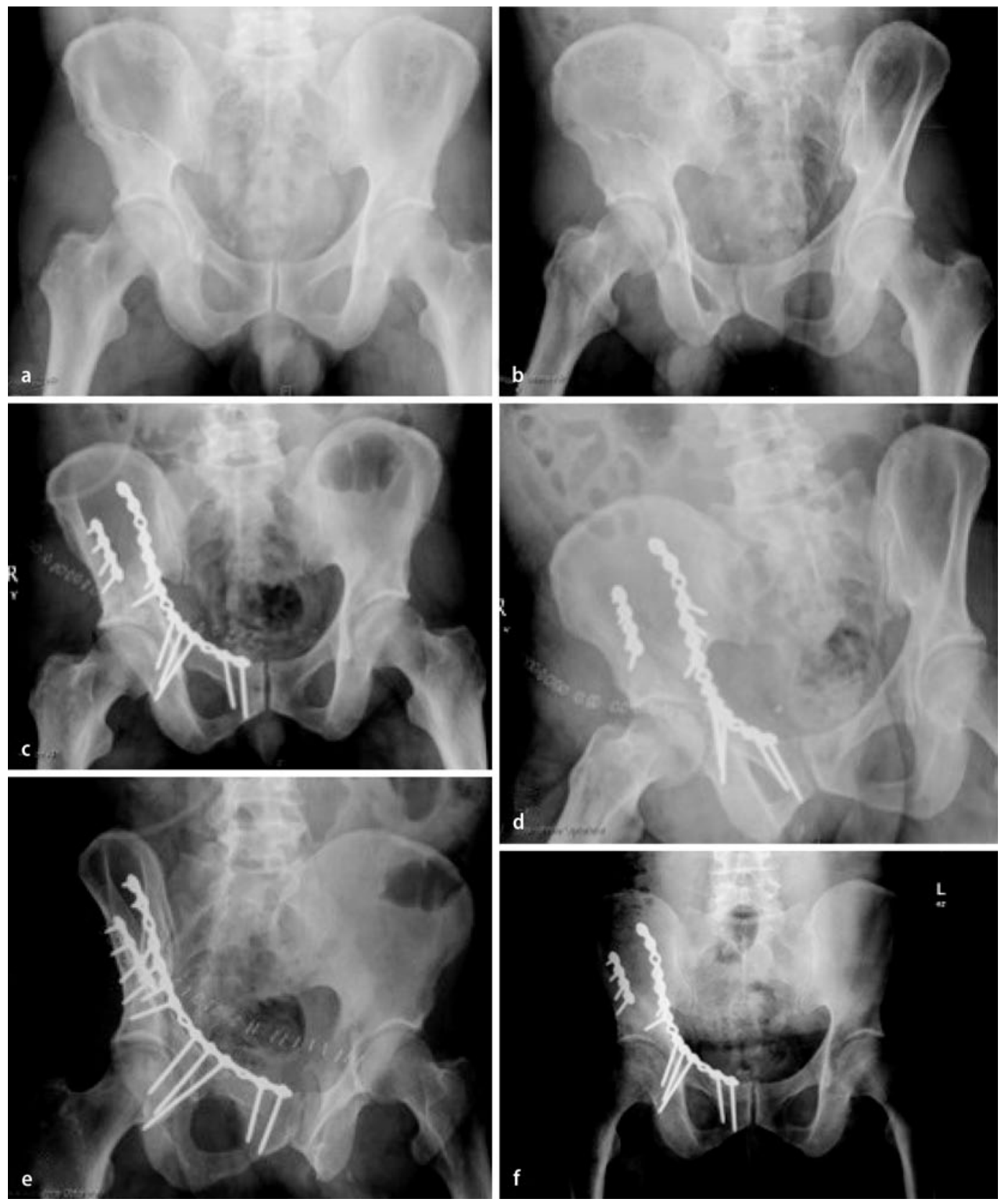

Abb. $6 \varangle$ Fallbeispiel 2, a,b 2-Pfeiler-Fraktur, c-e Reposition über ilioinguinalen Zugang, $\mathbf{f}$ regelrechter radiologischer Befund nach 2 Jahren

grund des ggf. verschleiernden Rechenalgorithmus zur Oberflächenanalyse nicht zu empfehlen.

Dislozierte Frakturen und Frakturen mit fehlender Gelenkkongruenz sollten operativ versorgt werden. Extensionsbehandlungen haben heutzutage im Wesentlichen nur noch historische Bedeutung, die Repositionsmöglichkeiten und insbesondere die Retention der Gelenkfragmente sind ungenügend. Da die operative Rekonstruktion einen hohen chirurgischen und infrastrukturellen Aufwand darstellt und schon bei verbleibender Stufenbildung $>2 \mathrm{~mm}$ und Spaltaus- prägung >3-4 mm mit einer deutlich ansteigenden Arthroserate zu rechnen ist, sollte jeder Eingriff sehr gut geplant werden. Gegebenenfalls ist die Kontaktaufnahme mit einem spezialisierten Zentrum für die Prognose des Patienten vorteilhafter.

\section{Planung und Durchführung}

Die korrekte Klassifikation erlaubt in der Regel bereits eine zuverlässige Zugangswahl. Trotz der vielen in der Literatur beschriebenen Zugänge werden heutzutage im Standardverfahren nur noch
- der Kocher-Langenbeck-Zugang (KL) und

- der ilioinguinale Zugang

angewendet. Ersterer erfuhr durch die Erweiterung mit der Trochanter-Flip-Osteotomie in Seitenlage [3] einen deutlichen Zugewinn. Die Möglichkeit der therapeutischen Hüftluxation verbessert die Einsicht in das Gelenk dramatisch, allerdings sind die Repositionsmöglichkeiten des vorderen Pfeilers noch eingeschränkt.

Der ilioinguinale Zugang erfährt durch die Zunahme der geriatrischen $\mathrm{Pa}$ tienten immer weitere Verbreitung, auch 


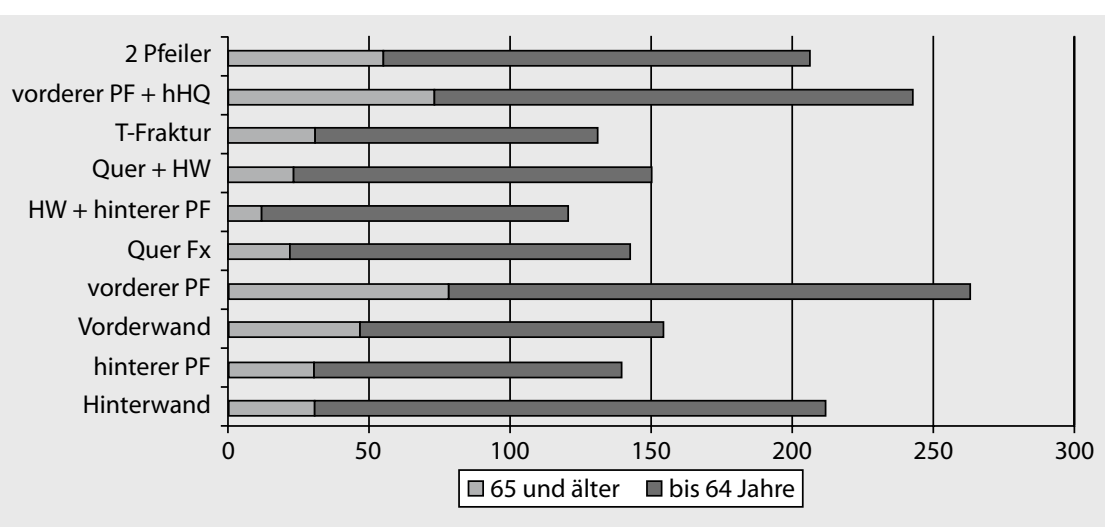

Abb. 7 வ Übersicht der AG (Arbeitsgemeinschaft) Becken über die Häufigkeit von Azetabulumfrakturen im Alter ( $n=1911$, Zeitraum: 1991-2007), 2 Pfeiler 2-Pfeiler-Frakturen, Fx Fraktur, $h H Q$ hintere Hemiquerfraktur, $H W$ hintere Wand, PF Pfeiler, $T$ T-Fraktur

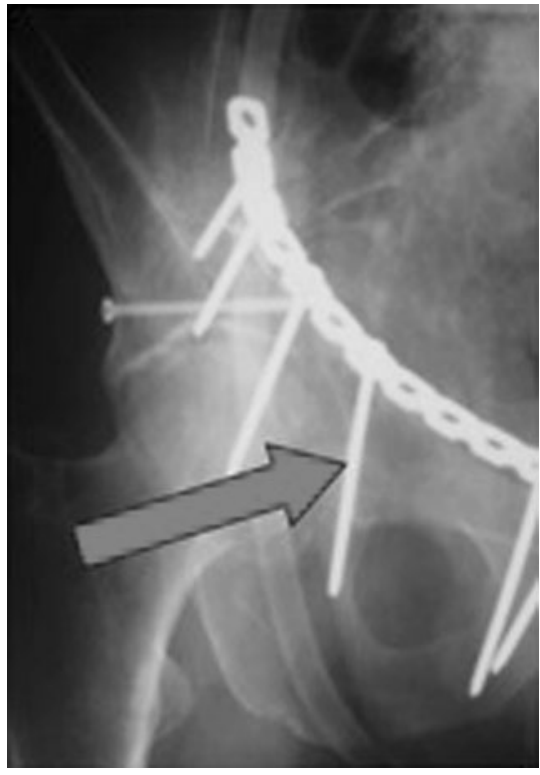

Abb. $8 \Delta$ Beispiel einer quadrilateralen Schraube nach Letournel

hier sind Modifikationen, wie die alleinige Benutzung des sog. 3. und 1. Fensters beschrieben (sog. Stoppa-Zugang, [1]).

Erweiterte Zugänge treten immer mehr in den Hintergrund und werden an sich nur noch für Spätrekonstruktionen in Ausnahmefällen eingesetzt.

Trotz der zunehmenden operativen Erfahrung handelte es sich bei operativen Maßnahmen am Azetabulum um große Eingriffe, bei denen es potenziell auch zu lebensbedrohlichen Situationen kommen kann. Eine gute Infrastruktur wie Blutbank, Cellsaver, Intensivstation usw. müssen neben einer ausreichenden ope rativen Erfahrung des Teams vorhanden sein. Spezielle Instrumentarien sind nö- tig, für die anatomischen Besonderheiten und die ggf. hohen aufzubringenden Kräfte sind Grundinstrumentarien nicht ausreichend!

Der Einsatz der Navigation kann sinnvoll sein, aber auf keinen Fall fundierte anatomische Kenntnisse ersetzen! Vorteile bietet eine intraoperative 3D-Bildgebung der neuesten Generation, sie erlaubt in der Regel eine zuverlässige Kontrolle der Repositionsqualität und schließt intraartikuläre Implantatlagen aus.

Kritisch soll in diesem Zusammenhang auf die sich schnell verbreitende Indikation zur navigationsunterstützten Verschraubung unverschobener Azetabulumfrakturen hingewiesen werden. In der Regel bedürfen diese keiner Stabilisierung, eine Frühmobilisation ist auch ohne Verschraubung problemlos möglich. Da bei jedem Eingriff immer die Gefahr einer dann für das Gelenk deletären intraartikulären Implantatlage droht, sollten derartige Eingriffe auf die sehr seltenen Indikationen einer wenig verschobenen, aber potenziell dislokationsgefährdeten Fraktur eingeschränkt werden.

Im Folgenden sind typische, aktuelle Versorgungsbeispiele dargestellt.

Fallbeispiel 1. Der 41-jährige Patient hatte sich im Rahmen eines PKW-Unfalls die in - Abb. 5 dargestellte Verletzung zugezogen (Querfraktur und hintere Wand, - Abb. 5a-c) und wurde uns zur operativen Versorgung zu verlegt. Nach Reposition über den KL-Zugang (• Abb.5d) konnte unmittelbar postoperativ mit der Mobilisation begonnen werden, in der
2-Jahres-Kontrolle zeigte sich der Patient klinisch beschwerdefrei bei regelrechtem radiologischem Befund (- Abb. 5e).

Fallbeispiel 2. Der junge Patient hatte sich im Rahmen eines Sturzes eine 2-Pfeiler-Fraktur (• Abb. 6a,b) zugezogen. Nach Klassifikation und operativer Planung erfolgte die Reposition über den ilioinguinalen Zugang (• Abb. 6ce). Nach zeitgerechter Mobilisation wurde der Patient zügig in die Rehabilitation entlassen und zeigte sich in der Kontrolle nach 2 Jahren beschwerdefrei bei regelrechtem radiologischem Befund.

\section{Azetabulumfrakturen bei geriatrischen Patienten}

Während noch vor 20 Jahre die Operation von Azetabulumfrakturen bei verminderter Knochenqualität eine klare Kontraindikation darstellte, machte deren rasch zunehmende Zahl bei geriatrischen Patienten ein Umdenken notwen$\operatorname{dig}$ (• Abb.7). In der Regel handelt es sich um rüstige Patienten mit teilweise hohem körperlichem Anspruch und Aktivitätsverhalten. Typischerweise treten die Frakturen nach Stürzen auf das gestreckte Bein auf. Die resultierenden Bruchtypen sind daher gehäuft

- Frakturen des vorderen Pfeilers,

- Frakturen des vorderen Pfeilers mit hinterer Hemiquerfraktur und 2-Pfeiler-Frakturen.

Diese Frakturformen zeichnen sich durch eine Instabilität des Domfragments aus. Der primäre Einsatz der Hüftendoprothetik ist daher limitiert, da nur in Verbindung mit einer ausreichenden Stabilisierung des vorderen Pfeilers eine sichere Pfannenverankerung erreicht werden könnte.

Im eigenen Vorgehen wurden daher zunächst unter dem Ansatz eines zweizeitigen Vorgehens eine Reposition und Stabilisierung über den ilioinguinalen Zugang begonnen. Die ersten Ergebnisse zeigten schnell, dass sowohl Repositionsqualität als auch die Stabilisierungsmöglichkeiten so gut waren, dass ein prothetischer Ersatz nur in wenigen Fällen erforderlich war. Die 2-Jahres-Ergebnisse entsprechen sogar in etwa denen jüngerer 


\section{Becken, Acetabulum, hüftgelenksnahe Frakturen}
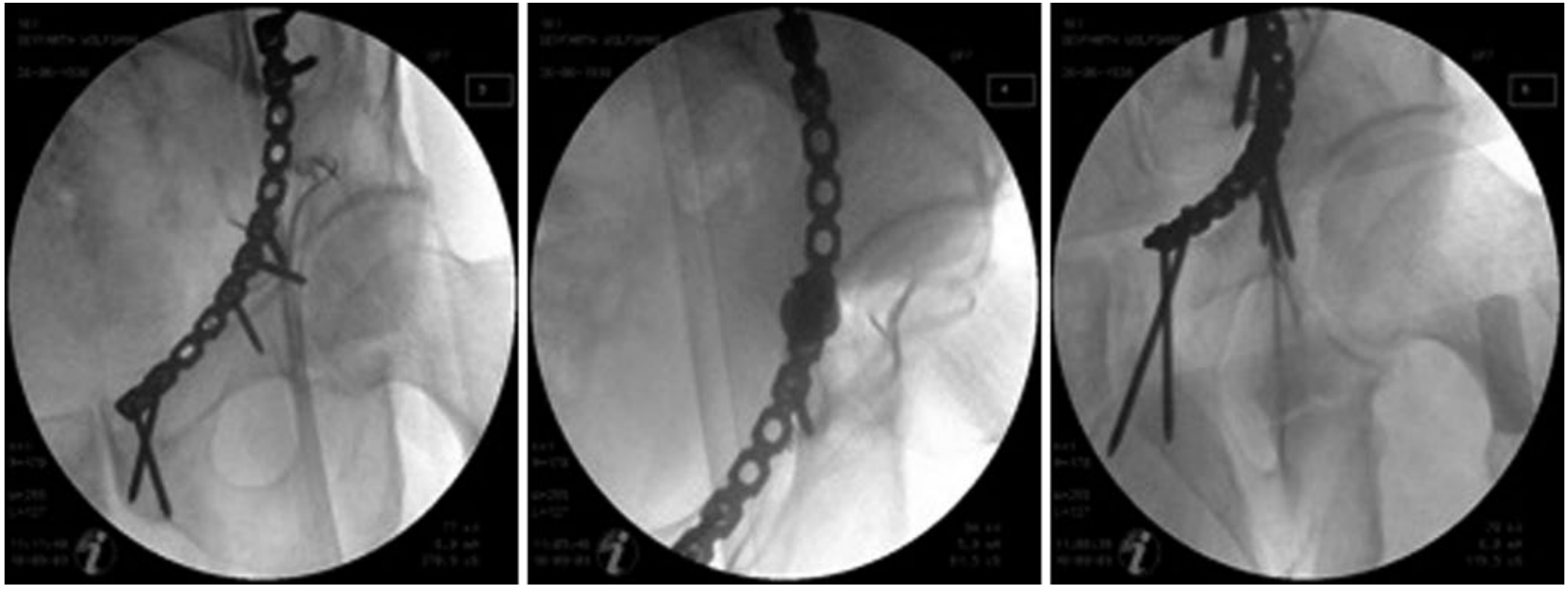

Abb. $9 \Delta$ Beispiel der intraoperativen Lagekontrolle der eingebrachten Schrauben, Dokumentation der extraartikularen Lage der Schrauben mittels tangentialer und axialer Projektionen
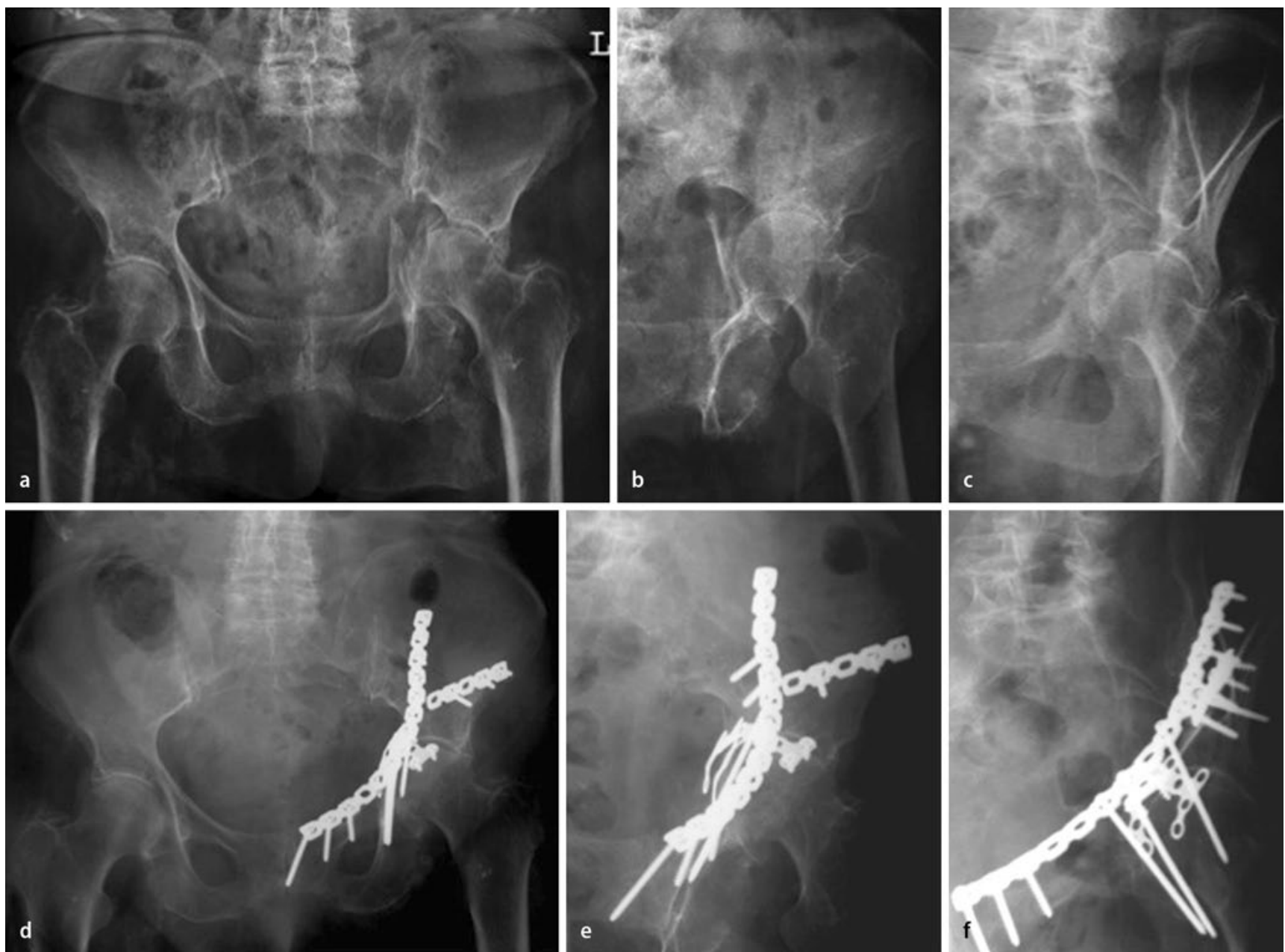

b

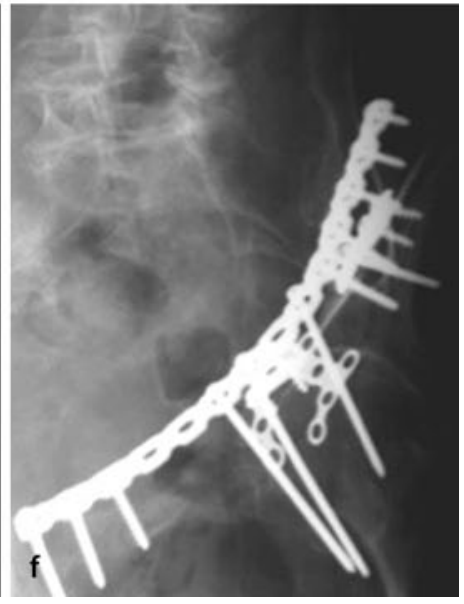

Abb. $10 \Delta$ Fallbeispiel 3: a-c Nativröntgenaufnahmen der Verletzung (tiefe Fraktur, vorderer Pfeiler und hintere Hemiquer-

fraktur), $\mathbf{d - f}$ anatomische Reposition 

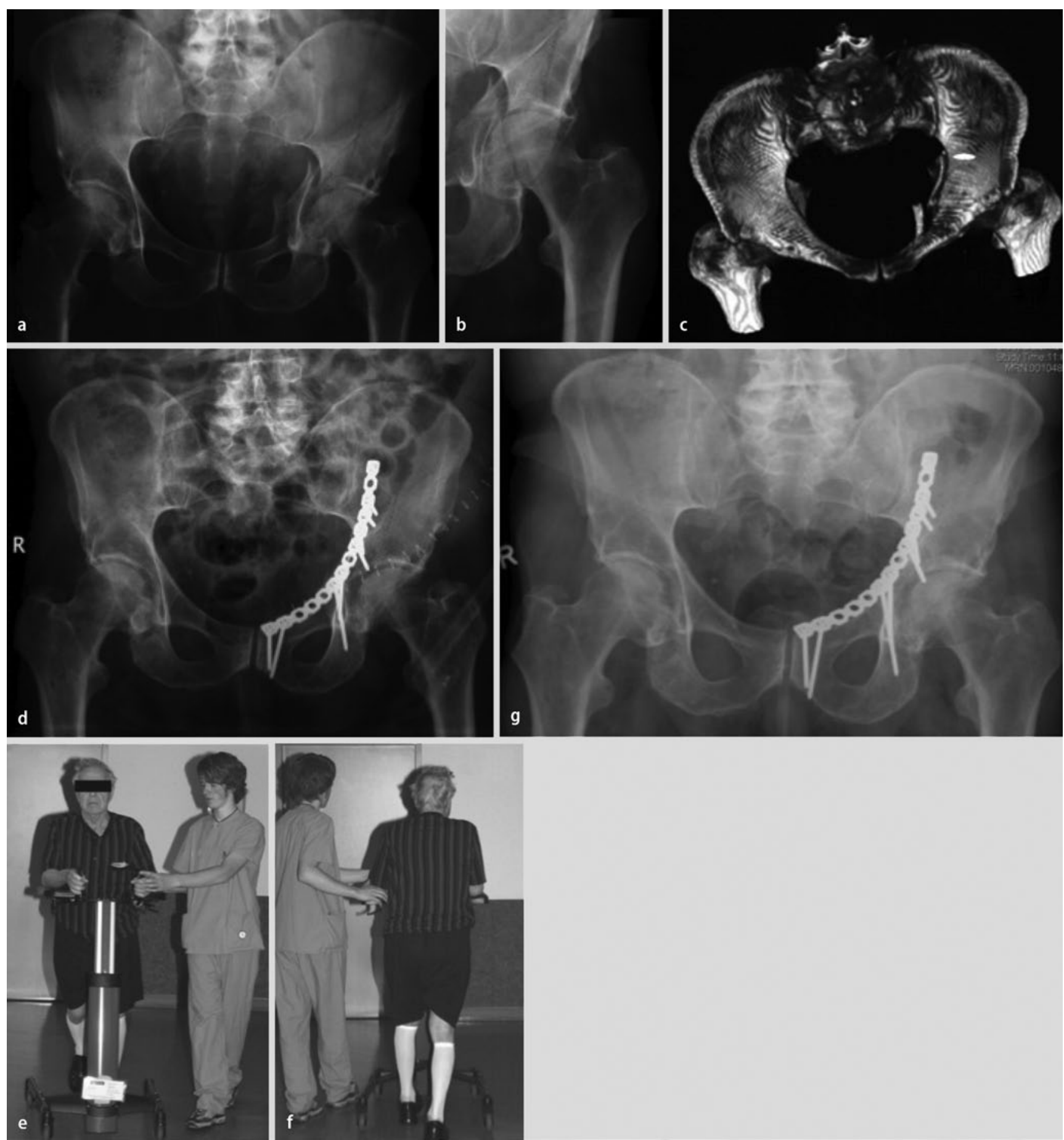

Abb. $11 \Delta$ Fallbeispiel 4: a-c Azetabulumfraktur (vorderer Pfeiler und hintere Hemiquerfraktur), d operative Stabilisierung über den ilioinguinalen Zugang, e,f Mobilisation bereits am 4. postoperativen Tag, $\mathbf{g}$ vollständige Rehabilitation bei 2-JahresKontrolle

Patienten [9]. Spezielle Implantate sind in der eigenen Erfahrung nicht notwendig, eine Schlüsselrolle nimmt aber eine absolut stabile Fixation zwischen vorderem und hinterem Pfeiler ein. Dazu wird die sog. quadrilaterale Schraube nach Letournel distal des Gelenks, etwa vom Tu- berculum iliopectinea startend, sehr nahe an der quadrilateralen Fläche anliegend bis in das Tuber ischiadicum eingebracht (- Abb. 8). Mit dem Widerlager der Platte hat diese Schraube eine ausgezeichnete biomechanische Funktion und inhibiert zuverlässig eine Zentralisation des Kopfs durch Verhinderung des Auseinanderweichens der Gelenkfragmente [2]. Eine exakte intraoperative Kontrolle der Implantatlage ist jedoch unumgänglich, dabei sind insbesondere Projektionen axial und tangential zur Schraubenlage einzusetzen (• Abb. 9). 
Durch Einsatz dieser Techniken konnten zwischenzeitlich auch hochbetagte $\mathrm{Pa}$ tienten bis in das 10. Lebensjahrzehnt zuverlässig versorgt und mobilisiert werden (Fallbeispiele 3 und 4, $\bullet$ Abb. 10, 11).

Fallbeispiel 3. Die 94-jährige Patientin zog sich bei einem Sturz im Garten auf die linke Hüfte eine tiefe Fraktur des vorderen Pfeilers und eine hintere $\mathrm{He}$ miquerfraktur zu. Nach Klassifikation der Verletzung mit Hilfe der Nativröntgenaufnahmen (• Abb. 10a-c) und Planung der Operation wurde die Fraktur über einen ilioinguinalen Zugang stabilisiert. Die postoperativen Röntgenbilder zeigten eine achsengerechte Reposition (- Abb. 10d-f), und die Patientin konnte zeitgerecht mobilisiert werden.

Fallbeispiel 4. Ein 89-jähriger Patient hatte sich im Rahmen eines häuslichen Sturzes die in $\bullet$ Abb. 11a-c gezeigte Azetabulumfraktur (vorderer Pfeiler und hintere Hemiquerfraktur) zugezogen. Auch hier erfolgte nach Klassifikation und sorgfältiger Planung die operative Stabilisierung über den ilioinguinalen Zugang (- Abb. 11d). Der Patient konnte bereits am 4 . postoperativen Tag mobilisiert werden (- Abb. 11e,f) und war bei der 2-Jahres-Kontrolle ( $\bullet$ Abb. 11g) vollständigen rehabilitiert.

\section{Ausblick}

Die zuverlässige und Erfolg versprechende Versorgung von Azetabulumfrakturen erfordert umfassende Kenntnisse in der Anatomie, der Pathobiomechanik und den einzelnen spezifischen operativen Techniken. Mit Hilfe der deutlich verbesserten Bildgebung sollten komplette Fehleinschätzungen der Frakturtypen nicht mehr auftreten. Da aber gerade bei der operativen Versorgung eine optimale Reposition für das langfristige Outcome unumgänglich ist, sollten enge Kooperationen mit spezialisierten Zentren eingegangen werden, um die Prognose weiter zu verbessern.

\section{Korrespondenzadresse}

Prof. Dr. T. Pohlemann

Klinik für Unfall-, Hand-

und Wiederherstellungschirurgie,

Universitätsklinikum des Saarlandes, Kirrberger Straße, 66421 Homburg/Saar

tim.pohlemann@uks.eu

Interessenkonflikt. Der korrespondierende Autor weist für sich und seine Koautoren auf folgende Beziehungen hin:

Kein Interessenkonflikt mit der vorgestellten Thematik. Chairman AOTR-System, Vizepräsident der Deutschen

Gesellschaft für Unfallchirurgie

\section{Literatur}

1. Cole JD, Bolhofner BR (1994) Acetabular fracture fixation via a modified Stoppa limited intrapelvic approach. Description of operative technique and preliminary treatment results. Clin Orthop Relat Res 305:112-123

2. Culemann U, Marintschev I, Gras F, Pohlemann T (2011) Infra-acetabular corridor - technical tip for an additional screw placement to increase the fixation strength of acetabular fractures. JTrauma 70(1):244-246

3. Ganz R, Gill TJ, Gautier E et al (2001) Surgical dislocation of the adult hip a technique with full access to the femoral head and acetabulum without the risk of avascular necrosis. J Bone Joint Surg $\mathrm{Br}$ 83(8):1119-1124

4. Judet R, Judet J, Letournel E (1964) Fractures of the acetabulum: classification and surgical approaches for open reduction. Preliminary report. J Bone Joint Surg Am 46:1615-1646

5. Letournel E (1980) Acetabulum fractures: classification and management. Clin Orthop Relat Res 151:81-106

6. Matta JM, Anderson LM, Epstein $\mathrm{HC}$, Hendricks C (1986) Fractures of the acetabulum. A retrospective analysis. Clin Orthop Relat Res 205:230-240

7. Müller M (1990) AO manual on internal fixation, 3. Aufl. Springer, Berlin Heidelberg New York

8. Pohlemann T (2008) Orthopädie und Unfallchirurgie. Acetabulumfrakturen. Elsevier Urban \& Fischer, München Jena, S 962

9. Tosounidis G, Culemann U, Bauer M et al (2011) Acetabular fractures in the elderly. Outcome of open reduction and internal fixation. Unfallchirurg 114(8):655-662

10. Tscherne H, Pohlemann T (2004) Unfallchirurgie, Becken und Acetabulum. Springer, Berlin Heidelberg New York 